\title{
Transfer Penalty of Public Transport
}

\author{
Ting Wang ${ }^{1, a, *}$, Siping Ke $\mathrm{K}^{1, \mathrm{~b}}$ \\ ${ }^{1}$ Department of Traffic Engineering, School of Highway, Chang'an University, Xi'an 710064, \\ China \\ a397949273@qq.com, ${ }^{a}$ kesipingw@163.com
}

Keywords: transfer penalty, principal component analysis, Logit model, public transport

\begin{abstract}
The public transport accounts for a large proportion in urban traffic, however, generally, travelers are unable to complete the trip with a single form of public transport. With the rapid development of the intelligent transportation and the application of big data, the accuracy of the generalized traffic time is more and more important to the passenger flow forecasting and service level optimization of public transportation system. An improved Logit model based on the theory of principal component analysis is established to calibrate the transfer penalty, which can cover the correlation among the variables. The calibration results show that the improved model is more accurate than the traditional Logit model, the conclusion is more reliable. In this paper, when the total time of the options is similar, if taking the different number of bus line into consideration, the transfer penalty is 12.13 minutes when the number of bus line is one, while it's 9.93 minutes when the number of bus line is two; if taking the same number of bus line into consideration, when there is only one bus line, the transfer penalty is 10.13 minutes, while it's 7.92 minutes when the number of bus line is two.
\end{abstract}

\section{Introduction}

With the implement of bus priority policy in China, public transport has been vigorously developed in recent years; public transport plays a more and more important role in urban traffic, but travelers rarely can directly reach destinations, most time the travel is the combination of subway and bus. More and more accurate generalized travel time is necessary because of the rapid development of intelligent transportation, the application of traffic big data, the more accurate passenger flow forecast and the optimization of service level of public transportation system. However, the researches on transfer penalty are not many, so the research on the transfer penalty of public transport is particularly important.

The existing researches on the application of Logit model in traffic mainly focus on the mode choice and the calculation of transfer penalty. The utility theory in economics were introduced into the field of transportation in the 1970s and the mode choice from the perspective of disaggregation is studied ${ }^{[1]}$ (Ben-Akiva et al.). To analyze the influence of the new subway on the choice of traffic mode, a NL model was established based on the RP/SP survey ${ }^{[2]}$ (Cherchi et al.). Considering the individual choice preference, a Mixed Logit model was established to analyze the choice behavior of traffic mode ${ }^{[3]}$ (McFadden et al.). To calculate the transfer penalty values on different transfer modes, a Logit model was established based on the New York data ${ }^{[4]}$ (Liu et al.). A path preference Logit model was established based on the geographic information system technology and it was 
applied to the subway transport system in Boston to improve the estimated accuracy of the transfer penalty ${ }^{[5]}$ (Guo Z et al.).

To calculate the transfer penalty value, a Mixed Logit model was established based on the smart card data of Seoul Korea ${ }^{[6]}$ (Yoo G.S). A binary Logit model was established to calculate the transfer penalty values of different transfer modes based on the survey data of different transfer situations in the multi-mode public transport network of the urban rail transit and bus in Shanghai ${ }^{[7]}$ (Wang et al.). Bus transfer behaviors and characteristics in Melbourne, Australia were examined based on origin-destination survey data set ${ }^{[8]}$ (Currie et al.).

What should be pointed out is that the establishment of MNL model, NL model and Mixed Logit model need to ensure that there is no linear relationship among the variables. However, there is always linear relationship among the variables to some degree, and the linear relationship may influence the reliability of the model. In order to overcome the correlation among variables, an improved Logit model based on the theory of principal component analysis is established. Also, previous studies about transfer penalty of public transport have never taken the bus line number into consideration. Based on the survey data of bus transfer in Xi'an, the bus transfer penalty value with different number of bus line is calculated.

\section{Transfer Penalty}

Travelers usually can't complete the whole trip only using bus or subway, transfer is always necessary. When choosing transfer mode, walking time, waiting time, on-vehicle time and the number of optional bus line are important factors. The transfer penalty value is an extra psychological burden that travelers realize and generate in the transfer phase, but it does not belong to the travel time. It does not include the walking transfer time, the influence caused by the comfort, security or other objective environment, and it is a specific value.

The definition of transfer penalty will have some differences because of the researches, generally, the transfer penalty is defined as travelers' perception of transfer time (including transfer walking time and transfer waiting time) that is relative to the on-vehicle time. Assuming traveler n's perception of 6 minutes on-vehicle time is equal to the perception of 1 minute transfer time, then for traveler $\mathrm{n}$, the transfer time is 6 minutes on-vehicle time. Therefore, the transfer penalty can be defined as the ratio of the coefficient of transfer time to the coefficient of on-vehicle time in the utility function. The mathematical expression is shown as equation (1):

$$
T P=\alpha_{1} / \alpha_{2}
$$

Where TP represents transfer penalty, $\alpha_{1}$ represents the coefficient of transfer time in the utility function, $\alpha_{2}$ represents the coefficient of on-vehicle time in the utility function.

Previous studies on transfer penalty value have different results ${ }^{[9-16]}$ (Algers et al, Hunt, Liu et al, Wardman et al, Guo et al, Sun, Douglas et al, Gong et al ): one class focused on 2.5 to 4 minutes; another class focused on 8 to 10 minutes; some studies held that transfer penalty value is 1.8 to 3 times of the total transfer time; and a small part of the studies held that the transfer penalty value is larger than 30 minutes. This paper proposes an improved Logit model based on the theory of principal component analysis, further, taking the bus line number into account, the transfer penalty is calculated.

\section{Methodology}

\subsection{Basic Model}

Pursuing the maximum utility is a significant principle of disaggregate model, in which travelers 
are supposed to know all the information about alternatives and follow the compensation theory ${ }^{[17]}$ (Guan). The utility function $U_{i n}$ of traveler $\mathrm{n}$ for mode $i$ is shown in equation (2):

$$
U_{\text {in }}=V_{i n}+\varepsilon_{i n}
$$

Where $V_{i n}$ represents fixed utility; $\varepsilon_{i n}$ represents a random term.

Assuming the choice set for traveler $\mathrm{n}$ is $A_{n}$, and the utility of mode $j$ is $U_{j n}$, then the condition that traveler $n$ selects mode $i$ from $A_{n}$ is shown in equation (3):

$$
U_{i n}>U_{j n}, i \neq j, j \in A_{n}
$$

The probability that mode $i$ is selected is shown in equation (4):

$$
P_{i n}=\frac{e^{V_{i n}}}{\sum_{j \in A_{n}} e^{V_{j n}}}=\frac{e^{\alpha_{i}+\beta_{i} X_{i}}}{\sum_{j \in A_{n}} e^{\alpha_{j}+\beta_{j} X_{j}}},\left(i=A_{n}\right)
$$

Where $P_{\text {in }}$ represents the probability that traveler n chooses mode $i ; \alpha_{i}$ represents the constant, $X_{i}$ represents the attribute; $\beta_{i}$ represents the coefficient of attribute $X_{i}$.

\subsection{Principal Component Analysis}

Principal component analysis is a data dimension reduction algorithm, and also a feature extraction method ${ }^{[18]}$ (Jollife). It improves the anti-jamming capability of the raw data and reorganizes the original indicators with a certain correlation to form a new set of indicators that are irrelevant to each other. These new indicators are linear combination of original indicators.

The main idea of principal component analysis is to express the relationship using the selected linear combination variance. The larger the $\operatorname{Var}\left(F_{1}\right)$ is, the more information $F_{1}$ contains. So, $F_{1}$ that is called the first principal component should have the largest variance. If $F_{1}$ cannot represent the original indicators, the second linear combination $F_{2}$ should be considered, also, the existing information in the $F_{1}$ should be removed in $F_{2}$, thus data redundancy can effectively be reduced and original information can be better reflected, namely $\operatorname{Cov}\left(F_{1}, F_{2}\right)=0$ should be satisfied. $F_{2}$ is called the second principal component, and based on this method, more principal components can be constructed until original indicators can be represented. Thus, the resulting principal components are not only irrelevant, but their variance decreases in turn.

Assuming that there are $\mathrm{n}$ samples with $\mathrm{p}$ variables to be observed in data set $\mathrm{X}$, the mathematical expression of the principal component analysis is shown in equation (5).

$$
X=\left[\begin{array}{ccc}
x_{11} & \vdots & x_{1 p} \\
\vdots & \ddots & \vdots \\
x_{n 1} & \cdots & x_{n p}
\end{array}\right]=\left[x_{1}, x_{2}, \cdots \cdots x_{p}\right]
$$

The principal component analysis is to combine the original $\mathrm{p}$ variables $x_{1}, x_{2}, \cdots \cdots x_{p}$, and the formed p new variables are shown in equation (6).

$$
\left\{\begin{array}{c}
F_{1}=w_{11} x_{1}+w_{21} x_{2}+\cdots+w_{p 1} x_{p} \\
F_{2}=w_{12} x_{1}+w_{22} x_{2}+\cdots+w_{p 2} x_{p} \\
\cdots \\
F_{p}=w_{1 p} x_{1}+w_{2 p} x_{2}+\cdots+w_{p p} x_{p}
\end{array}\right.
$$


The coefficient $w_{i j}$ in the above models satisfies the following three conditions: $F_{i}$ and $F_{j}$ $(i \neq j ; j=1,2, \ldots \ldots p)$ are not related; The variance of $F_{1}, F_{2}, F_{3}, \cdots \cdots, F_{p}$ decreases in turn; $w_{k 1}^{2}+w_{k 2}^{2}+\cdots+w_{k p}^{2}=1, \quad k=1,2, \ldots \ldots, p$.

The new obtained random variables are irrelevant to each other and the variance decreases sequentially when the above three conditions are satisfied. If the former $\mathrm{k}$ principal components contain $85 \%$ and more of the total variance, it indicates that the former $\mathrm{k}$ principal components can retain the original variable information to the utmost.

\subsection{Improved Model}

First, the original $i$ variables $X_{1}, X_{2}, \cdots, X_{n}$ were analyzed using the principal component analysis, and then the principal components $F_{1}, F_{2}, \cdots, F_{K}$ were filtered when the cumulative contribution rate reaches $85 \%$ and more of total variance. The correlation coefficients among the principal components are zero. Finally, the improved Logit model is established with the resulting principal components, and the expression is shown in equation (7).

$$
P_{i n}=\frac{e^{V_{i n}}}{\sum_{j \in A_{n}} e^{V_{j n}}}=\frac{e^{\alpha_{i}+\beta_{i} F_{i}}}{\sum_{j \in A_{n}} e^{\alpha_{j}+\beta_{j} F_{j}}},\left(i=A_{n}\right)
$$

\section{Data Collection}

Personal attributes and mode attributes are selected to design the questionnaire, and personal attributes and levels are shown in Table 1.

Table 1 Personal attributes

\begin{tabular}{|c|c|c|}
\hline Attribute category & Attributes & Attribute levels \\
\hline \multirow{9}{*}{ Personal attributes } & \multirow{2}{*}{ Gender } & Male \\
\hline & & Female \\
\hline & \multirow{4}{*}{ Age } & Age of $18-30$ \\
\hline & & Age of $31-40$ \\
\hline & & Age of $41-50$ \\
\hline & & Age above 50 \\
\hline & \multirow{3}{*}{ Education level } & Under high school \\
\hline & & University \\
\hline & & Master and above \\
\hline
\end{tabular}

Mode attributes are: subway travel time, bus travel time, transfer walking time and transfer waiting time. If the bus line number is different, the attribute values will be different, so two different values are used in the level setting.

In this paper, SP survey was adopted to obtain the data, which is an investigation under the condition of hypothetical scenario. Pre-determined influence factors and levels are combined into different scenarios for travelers to compare, order or make discrete choice, and the choice behaviors that respondents prefer to adopt are obtained.

To avoid the impact that the total travel time of different transfer programs have on travelers' choices, the total travel time is set to the similar value. Simultaneously, the balanced design method is introduced to ensure the survey accuracy and avoid the long-term response. The final scenario combinations are shown in Table 2. 
Table 2 Scenario combinations

\begin{tabular}{|c|c|c|c|c|c|c|}
\hline Scenarios & Choice & Bus line number & Subway travel time & Walking time & Waiting time & Bus travel time \\
\hline \multirow{2}{*}{ Scenario1 } & A & 1 & 10 & 6 & 11 & 29 \\
\hline & $\mathrm{B}$ & 2 & 25 & 9 & 4 & 13 \\
\hline \multirow{2}{*}{ Scenario2 } & A & 1 & 15 & 12 & 5 & 23 \\
\hline & B & 2 & 20 & 2 & 10 & 18 \\
\hline \multirow{2}{*}{ Scenario 3} & A & 1 & 20 & 3 & 14 & 17 \\
\hline & B & 2 & 15 & 12 & 1 & 23 \\
\hline \multirow{2}{*}{ Scenario 4} & A & 1 & 25 & 9 & 8 & 11 \\
\hline & B & 2 & 10 & 6 & 7 & 28 \\
\hline
\end{tabular}

When the optional bus line number is same: bus line number is one and two, the transfer penalties are calculated, and the scenario combination designs are shown in Table 3 and Table 4 respectively.

Table 3 Scenario combinations with bus line number is one

\begin{tabular}{|c|c|c|c|c|c|c|}
\hline Scenarios & Choice & Bus line number & Subway travel time & Walking time & Waiting time & Bus travel time \\
\hline \multirow{2}{*}{ Scenario 1} & A & 1 & 10 & 6 & 11 & 29 \\
\hline & $\mathrm{B}$ & 1 & 25 & 9 & 8 & 11 \\
\hline \multirow{2}{*}{ Scenario 2} & A & 1 & 15 & 12 & 5 & 23 \\
\hline & $\mathrm{B}$ & 1 & 20 & 2 & 14 & 17 \\
\hline \multirow{2}{*}{ Scenario 3} & A & 1 & 20 & 3 & 14 & 17 \\
\hline & $\mathrm{B}$ & 1 & 15 & 12 & 5 & 23 \\
\hline \multirow{2}{*}{ Scenario 4} & A & 1 & 25 & 9 & 8 & 11 \\
\hline & B & 1 & 10 & 6 & 11 & 29 \\
\hline
\end{tabular}

Table 4 Scenario combinations with bus line number is two

\begin{tabular}{|c|c|c|c|c|c|c|}
\hline \multirow{2}{*}{ Scenarios } & Choice & Bus line number & Subway travel time & Walking time & Waiting time & Bus travel time \\
\hline \multirow{2}{*}{ Scenario 1 } & A & 2 & 10 & 6 & 7 & 28 \\
\cline { 2 - 7 } & B & 2 & 25 & 9 & 4 & 13 \\
\hline \multirow{2}{*}{ Scenario 2 } & A & 2 & 15 & 2 & 12 & 23 \\
\cline { 2 - 7 } & B & 2 & 20 & 12 & 1 & 18 \\
\hline \multirow{2}{*}{ Scenario 3 } & A & 2 & 15 & 9 & 4 & 23 \\
\cline { 2 - 7 } & B & 2 & 25 & 6 & 7 & 13 \\
\cline { 2 - 7 }
\end{tabular}

In this survey 350 persons were asked and 80 percent of the data is valid. Because there are three scenario combinations and in each scenario combination there are four scenarios, 1120 valid data was obtained for each scenario combination.

\section{Result Analysis}

The traditional Logit model and the improved Logit model are established based on the 1120 valid data taking the different number of bus line into consideration. The calibration results of the 
traditional Logit model are shown in Table 5.

Table 5 Calibration results of traditional Logit model

\begin{tabular}{|c|c|}
\hline Attributes & Calibration coefficient (t test) \\
\hline Constant & $-1.5223(-3.5106)$ \\
\hline Age of 31-40 & $0.1123(3.0126)$ \\
\hline On-vehicle time & $-0.1303(-2.0359)$ \\
\hline Waiting time & $-0.1626(-3.1327)$ \\
\hline Walking time & $-0.1808(-5.2936)$ \\
\hline Bus line number & $0.5726(2.6537)$ \\
\hline$\rho^{2}$ & 0.2031 \\
\hline
\end{tabular}

The calibration results show that the significant factors are age of 31-40, on-vehicle time, walking time, waiting time and the number of optional bus line number.

The principal component analysis of one transfer bus number is carried out except for the attribute of bus number. The results and the principal component tables are shown in Table 6, Table 7 respectively.

Table 6 Results of the principal component analysis

\begin{tabular}{|c|c|c|c|c|c|c|}
\hline \multirow{2}{*}{ Component } & \multicolumn{3}{|c|}{ Initial eigenvalue } & \multicolumn{3}{c|}{ Extraction factor } \\
\cline { 2 - 7 } & \multirow{2}{*}{ Sum } & Variance \% & Contribution rate\% & \multirow{2}{*}{ Sum } & \multirow{2}{*}{ Variance \% } & Contribution rate\% \\
\hline 1 & 2.001 & 16.677 & 16.677 & 2.001 & 16.677 & 16.677 \\
\hline 2 & 2.000 & 16.668 & 33.345 & 2.000 & 16.668 & 33.345 \\
\hline 3 & 1.893 & 15.774 & 49.119 & 1.893 & 15.774 & 49.119 \\
\hline 4 & 1.446 & 12.047 & 61.166 & 1.446 & 12.047 & 61.166 \\
\hline 5 & 1.421 & 11.838 & 73.004 & 1.421 & 11.838 & 73.004 \\
\hline 6 & 1.184 & 9.871 & 82.874 & 1.184 & 9.871 & 82.874 \\
\hline 7 & 1.015 & 8.462 & 91.336 & 1.015 & 8.462 & 91.336 \\
\hline 8 & 0.913 & 7.612 & 98.948 & & & \\
\hline
\end{tabular}

Table 7 Principal component table with bus number is one

\begin{tabular}{|c|c|c|c|c|c|c|c|}
\hline \multirow{2}{*}{ Attributes } & \multicolumn{9}{|c|}{ Principal component } \\
\cline { 2 - 8 } & 1 & 2 & 3 & 4 & 5 & 6 & 7 \\
\hline Gender & 0.009 & -0.001 & -0.055 & 0.079 & 0.228 & 0.070 & 0.629 \\
\hline Age of 18-30 & -0.005 & -0.015 & -0.133 & -0.531 & -0.286 & 0.067 & -0.134 \\
\hline Age of 31-40 & -0.002 & 0.004 & -0.003 & 0.079 & 0.651 & -0.103 & -0.278 \\
\hline Age of 41-50 & 0.004 & 0.003 & 0.112 & 0.346 & -0.315 & -0.520 & 0.298 \\
\hline Age above 50 & 0.005 & 0.008 & 0.002 & 0.213 & -0.083 & 0.716 & 0.272 \\
\hline Under high school & 0.010 & 0.038 & 0.400 & 0.194 & -0.114 & 0.163 & -0.367 \\
\hline University & -0.016 & -0.055 & -0.510 & 0.144 & -0.027 & -0.058 & -0.024 \\
\hline Master and above & 0.010 & 0.034 & 0.261 & -0.417 & 0.164 & -0.102 & 0.444 \\
\hline Subway travel time & -0.362 & -0.032 & -0.023 & -0.002 & -0.007 & -0.008 & -0.012 \\
\hline Waiting time & -0.344 & -0.162 & -0.013 & -0.003 & -0.004 & -0.004 & -0.003 \\
\hline Walking time & -0.324 & -0.172 & -0.014 & -0.004 & -0.005 & -0.004 & -0.003 \\
\hline Bus travel time & -0.362 & -0.032 & -0.023 & -0.002 & -0.007 & -0.008 & -0.012 \\
\hline
\end{tabular}

The principal component analysis of two transfer bus number is carried out except for the attribute of bus number. The results and the principal component tables are shown in Table 8, Table 9 respectively. 
Table 8 Results of the principal component analysis

\begin{tabular}{|c|c|c|c|c|c|c|}
\hline \multirow{2}{*}{ Component } & \multicolumn{3}{|c|}{ Initial eigenvalue } & \multicolumn{3}{c|}{ Extraction factor } \\
\cline { 2 - 7 } & Sum & Variance \% & Contribution rate\% & Sum & Variance \% & Contribution rate\% \\
\hline 1 & 2.030 & 16.913 & 16.913 & 2.030 & 16.913 & 16.913 \\
\hline 2 & 1.970 & 16.415 & 33.328 & 1.970 & 16.415 & 33.328 \\
\hline 3 & 1.893 & 15.772 & 49.100 & 1.893 & 15.772 & 49.100 \\
\hline 4 & 1.446 & 12.047 & 61.147 & 1.446 & 12.047 & 61.147 \\
\hline 5 & 1.421 & 11.838 & 72.985 & 1.421 & 11.838 & 72.985 \\
\hline 6 & 1.184 & 9.871 & 82.855 & 1.184 & 9.871 & 82.855 \\
\hline 7 & 1.015 & 8.462 & 91.317 & 1.015 & 8.462 & 91.317 \\
\hline 8 & 0.913 & 7.612 & 98.929 & & & \\
\hline 9 & 0.126 & 1.052 & 99.981 & & & \\
\end{tabular}

Table 9 Principal component table with bus number is two

\begin{tabular}{|c|c|c|c|c|c|c|c|}
\hline \multirow{2}{*}{ Attributes } & \multicolumn{9}{|c|}{ Principal component } \\
\cline { 2 - 8 } & 1 & 2 & 3 & 4 & 5 & 6 & 7 \\
\hline Gender & 0.005 & 0.007 & -0.055 & 0.079 & 0.228 & 0.070 & 0.629 \\
\hline Age of 18-30 & -0.013 & 0 & -0.133 & -0.531 & -0.286 & 0.067 & -0.134 \\
\hline Age of 31-40 & 0.002 & -0.003 & -0.003 & 0.079 & 0.651 & -0.103 & -0.278 \\
\hline Age of 41-50 & 0.003 & 0.004 & 0.112 & 0.346 & -0.315 & -0.520 & 0.298 \\
\hline Age above 50 & 0.009 & 0 & 0.003 & 0.213 & -0.083 & 0.716 & 0.272 \\
\hline Under high school & 0.029 & -0.002 & 0.401 & 0.194 & -0.114 & 0.163 & -0.367 \\
\hline University & -0.044 & 0.004 & -0.512 & 0.144 & -0.027 & -0.058 & -0.024 \\
\hline Master and above & 0.028 & -0.003 & 0.262 & -0.417 & 0.164 & 0.102 & 0.444 \\
\hline Subway travel time & -0.481 & -0.106 & -0.016 & -0.002 & -0.006 & -0.008 & -0.012 \\
\hline Waiting time & -0.127 & -0.125 & -0.014 & -0.003 & -0.004 & -0.004 & -0.003 \\
\hline Walking time & -0.137 & -0.136 & -0.014 & -0.002 & -0.005 & -0.004 & -0.002 \\
\hline Bus travel time & -0.481 & -0.106 & -0.016 & -0.002 & -0.006 & -0.008 & -0.012 \\
\hline
\end{tabular}

Based on the principal component table, the principal component data $F_{11}, F_{12}, F_{13}, F_{14}, F_{15}, F_{16}, F_{17}$ of one optional bus line and the principal component data $F_{21}, F_{22}, F_{23}, F_{24}, F_{25}, F_{26}, F_{27}$ of two optional bus line can be obtained by the corresponding deformed survey data. The improved Logit model is established based on the obtained data, and the calibration results of the model are shown in Table 10.

Table 10 Calibration results of the improved Logit model

\begin{tabular}{|c|c|}
\hline Attributes & Calibration coefficient (t test) \\
\hline Constant & $-1.8309(2.7532)$ \\
\hline Bus line number & $0.9219(3.0125)$ \\
\hline $\mathrm{F}_{12}$ & $0.9126(3.3152)$ \\
\hline $\mathrm{F}_{22}$ & $0.9621(2.8532)$ \\
\hline $\mathrm{F}_{13}$ & $6.3127(3.1562)$ \\
\hline $\mathrm{F}_{23}$ & $6.9815(2.9631)$ \\
\hline $\mathrm{F}_{16}$ & $-0.6283(-3.2109)$ \\
\hline $\mathrm{F}_{26}$ & $-0.8523(-2.8732)$ \\
\hline$\rho^{2}$ & 0.2952 \\
\hline
\end{tabular}

The comparing results of the significant influencing factor coefficients obtained by the traditional Logit model and the improved Logit model are shown in Table 11. 
Table 11 Results comparison

\begin{tabular}{|c|c|c|c|}
\hline Attributes & Traditional Logit model & $\begin{array}{c}\text { Improved Logit model } \\
\text { (bus line number is 1) }\end{array}$ & $\begin{array}{c}\text { Improved Logit model (bus } \\
\text { line number is 2) }\end{array}$ \\
\hline Constant & -1.5223 & 0.0618 & -2.0548 \\
\hline Age of 31-40 & 0.1123 & -0.1694 & -0.2069 \\
\hline On-vehicle time & -0.1303 & -0.2274 & -0.2146 \\
\hline Waiting time & -0.1626 & -0.2428 & -0.2252 \\
\hline Walking time & -0.1808 & \multicolumn{2}{|c|}{0.9219} \\
\hline Bus line number & 0.5726 & \multicolumn{2}{|c|}{0.2952} \\
\hline$\rho^{2}$ & 0.2031 & \multicolumn{2}{|c|}{} \\
\hline
\end{tabular}

It can be seen that the improved Logit model is more accurate than the traditional Logit model ( $\rho^{2}$ is larger), and the coefficient of the bus line number in the improved model is higher than the traditional Logit model. It can be concluded that the improved model is more reliable and closer to the actual situation. The results of the two models are standardized on the basis of on-vehicle time, and the results are shown in Table 12.

Table 12 Standardization results comparison

\begin{tabular}{|c|c|c|c|}
\hline Model & Attributes & Calibration coefficient & Standardization (minute) \\
\hline \multirow{4}{*}{ Traditional Logit model } & On-vehicle time & -0.1303 & 1.00 \\
\cline { 2 - 4 } & Waiting time & -0.1626 & 1.25 \\
\cline { 2 - 4 } & Walking time & -0.1808 & 11.68 \\
\cline { 2 - 4 } & Constant & -1.5223 & 1.00 \\
\hline \multirow{3}{*}{$\begin{array}{c}\text { Improved Logit model } \\
\text { (bus line number is 1) }\end{array}$} & On-vehicle time & -0.1694 & 1.34 \\
\cline { 2 - 4 } & Waiting time & -0.2274 & 1.43 \\
\cline { 2 - 4 } & Walking time & -0.2428 & 1.13 \\
\hline \multirow{3}{*}{$\begin{array}{c}\text { Improved Logit model } \\
\text { (bus line number is 2) }\end{array}$} & Constant & -2.0548 & 1.04 \\
\cline { 2 - 4 } & On-vehicle time & -0.2069 & 1.09 \\
\cline { 2 - 4 } & Waiting time & -0.2146 & 9.93 \\
\cline { 2 - 4 } & Walking time & -0.2252 & -2.0548 \\
\hline
\end{tabular}

As can be seen from the standardized results, in the traditional Logit model the transfer penalty, transfer penalty of walking time, transfer penalty of waiting time are 11.68 minutes, 1.39 minutes, 1.25 minutes respectively when the number of optional bus line is considered; in the improved Logit model when the bus line number is one, transfer penalty, transfer penalty of walking time, transfer penalty of waiting time are 12.13 minutes, 1.43 minutes, 1.34 minutes respectively; while they are 9.93 minutes, 1.09 minutes, 1.04 minutes respectively when the bus line number is two; moreover, models that consider the different number of optional bus line, the penalty values of them are between values of models that the bus line number is one and two. Also with the increase of bus number, the penalty values reduce.

Similarly, based on the survey data with the bus line number is one and two, the traditional Logit models and improved models are established, the comparing results are shown in Table 13, Table 14 and Table 15,Table 16 respectively.

Table 13 Comparing results with bus line number is one

\begin{tabular}{|c|c|c|}
\hline Attributes & Traditional Logit model ( $\mathrm{t}$ test) & Improved Logit model (t test) \\
\hline Constant & $-1.7862(-2.3685)$ & $-1.7026(-3.0521)$ \\
\hline Age of 31-40 & $0.1059(2.1863)$ & $0.0853(1.9702)$ \\
\hline On-vehicle time & $-0.1528(-2.8635)$ & $-0.1681(-3.2813)$ \\
\hline Waiting time & $-0.1762(-2.3715)$ & $-0.1853(-2.9613)$ \\
\hline Walking time & $-0.1951(-2.8519)$ & $-0.2016(-3.0216)$ \\
\hline$\rho^{2}$ & 0.2102 & 0.2869 \\
\hline
\end{tabular}


Table 14 Comparing results of the standardization dada

\begin{tabular}{|c|c|c|c|}
\hline Models & Attributes & Calibration coefficient & Standardization \\
\hline \multirow{4}{*}{ Traditional Logit model } & On-vehicle time & -0.1528 & 1.00 \\
\cline { 2 - 4 } & Waiting time & -0.1762 & 1.15 \\
\cline { 2 - 4 } & Walking time & -0.1951 & 1.28 \\
\cline { 2 - 4 } & Constant & -1.7862 & 11.69 \\
\hline \multirow{3}{*}{ The improved model } & On-vehicle time & -0.1681 & 1.00 \\
\cline { 2 - 4 } & Waiting time & -0.1853 & 1.10 \\
\cline { 2 - 4 } & Walking time & -0.2026 & 1.21 \\
\cline { 2 - 4 } & Constant & -1.7026 & 10.13 \\
\hline
\end{tabular}

Table 15 Comparing results with bus line number is two

\begin{tabular}{|c|c|c|}
\hline Attributes & Traditional Logit model (t test) & Improved Logit model (t test) \\
\hline Constant & $-1.6491(-2.4128)$ & $-1.4122(-2.1823)$ \\
\hline Age of 31-40 & $0.1231(2.1536)$ & $0.0968(1.9803)$ \\
\hline On-vehicle time & $-0.1635(-2.6105)$ & $-0.1783(-2.7519)$ \\
\hline Waiting time & $-0.1703(-2.1285)$ & $-0.1890(-2.3681)$ \\
\hline Walking time & $-0.1839(-2.3912)$ & $-0.2050(-2.2912)$ \\
\hline$\rho^{2}$ & 0.2042 & 0.3052 \\
\hline
\end{tabular}

Table 16 Comparing results of the standardization dada

\begin{tabular}{|c|c|c|c|}
\hline Models & Attributes & Calibration coefficient & Standardization \\
\hline \multirow{3}{*}{ Traditional Logit model } & On-vehicle time & -0.1635 & 1.00 \\
\cline { 2 - 4 } & Waiting time & -0.1703 & 1.04 \\
\cline { 2 - 4 } & Walking time & -0.1839 & 1.12 \\
\cline { 2 - 4 } & Constant & -1.6491 & 1.09 \\
\hline \multirow{3}{*}{ The improved model } & On-vehicle time & -0.1783 & 1.06 \\
\cline { 2 - 4 } & Waiting time & -0.1890 & 1.15 \\
\cline { 2 - 4 } & Walking time & -0.2050 & 7.92 \\
\cline { 2 - 4 } & Constant & -1.4122 & \\
\hline
\end{tabular}

The calculation results show that the improved model is more accurate than the traditional Logit model, so the results are more reliable. Also, the transfer penalty, the transfer penalty of walking time and the transfer penalty of waiting time are 10.13 minutes, 1.21 minutes and 1.10 minutes respectively, when the bus line number is one; while they are 7.92 minutes, 1.15 minutes and 1.06 minutes when the bus line number is two. The transfer penalty of walking time is bigger than the transfer penalty of waiting time, which indicates travelers are more sensitive to the walking time. With the increase of the bus line number, all the penalty values will decrease and the larger the degree of decline is, the bigger the impact is.

\section{Conclusions and Future Research}

To better serve the optimization of the bus system, more accurate generalized travel time is necessary. This paper takes the different number of bus line into consideration, which makes the bus transfer penalty more accurate. Previous studies have never taken the effect of the linear relationship among variables into consideration, to overcome the linear relationship, an improved Logit model based on the principal component theory is established. Based on the survey data in Xi'an, the traditional Logit model and the improved model are established respectively. The results show that the improved Logit model is more accurate than the traditional Logit model, so the transfer penalty is more reliable and closer to reality. In this paper, the total time of the options is similar, if taking the different number of bus line into consideration, when the number of bus line is one, the transfer penalty, the transfer penalty of walking time and the transfer penalty of waiting time are 12.13 minutes, 1.43 minutes and 1.34 minutes respectively; while they are 9.93 minutes, 1.09 minutes and 1.04 minutes when the number of bus line is two. If taking the same number of 
bus line into consideration, when there is only one bus line, the transfer penalty, the transfer penalty of walking time and the transfer penalty of waiting time are 10.13 minutes, 1.21 minutes and 1.10 minutes respectively; while they are 7.92 minutes, 1.15 minutes and 1.06 minutes when the number of bus line is two. Also, travelers are more sensitive to the walking time. With the increase of the bus line number all the penalty values will decrease.

In this paper, the bus punctuality rate and the traffic condition are not taken into consideration, how to quantify the factors that affect the reliability of the bus system when calculating the transfer penalty should be studied.

\section{References}

[1] Ben-Akiva, M., Palma, A. D., and Kanaroglou, P. (1984). Dynamic Model of Peak Period Traffic Congestion with Elastic Arrival Rates. Transportation Science, 20(3), 164-181.

[2] Cherchi, E., and Ortúzar, J. D. D. (2002). Mixed RP/SP Models Incorporating Interaction Effects. Transportation, 29(4), 371-395.

[3] Mcfadden, D., and Train, K. (2000). Mixed MNL Models for Discrete Response. Journal of Applied Econometrics, 15(5), 447-470.

[4] Liu, R., Pendyala, R., and Polzin, S. (1997). Assessment of Intermodal Transfer Penalties Using Stated Preference Data. Transportation Research Record Journal of the Transportation Research Board, 1607(1), 74-80.

[5] Guo, Z., and Wilson, N. (2004). Assessment of the Transfer Penalty for Transit Trips Geographic Information System-based Disaggregate Modeling Approach. Transportation Research Record Journal of the Transportation Research Board, 1872(1), 10-18.

[6] Yoo, G.S. (2015). Transfer Penalty Estimation with Transit Trips from Smartcard Data in Seoul, Korea. KSCE Journal of Civil Engineering, 19(4), 1108-1116.

[7] Wang, H., Wu, J.R. (2011) Transfer Penalty of Multi - mode Public Transport Network [C] China Urban Planning Annual Meeting. 2011.

[8] Currie, G., and Loader, C. (2010). Bus Network Planning for Transfers and the Network Effect in Melbourne, Australia. Transportation Research Record Journal of the Transportation Research Board, 2145(2145), 8-17.

[9] Algers, S., Hansen, S., and Tegner, G. (1975). Role of Waiting Time, Comfort, and Convenience in Modal Choice for Work Trip. Transportation Research Record, 38-51.

[10] Hunt, J. D. (1990). A Logit Model of Public Transport Route Choice. Ite Journal, 60(12), 26-30.

[11] Liu, R., Pendyala, R. M., and Polzin, S. (1998). Simulation of the Effects of Intermodal Transfer Penalties on Transit Use. Transportation Research Record Journal of the Transportation Research Board,1623(1), 88-95.

[12] Wardman, M., Hine, J., and Stradling, S. (2001). Interchange and Travel Choice - Volumes 1 and 2. Transport Research.

[13] Guo, Z., and Jr, J. F. (2008). Pedestrian Environments, Transit Path Choice, and Transfer Penalties: Understanding Land-use Impacts on Transit Travel. Environment and Planning B: Planning and Design,35(3), 461-479.

[14] Sun, Y. (2009). Transfer Penalty of Rail Transit Network [M].

[15] Douglas, N. J., and Jones, M. (2013, October). Estimating Transfer Penalties and Standardized Income Values of Time by Stated Preference Survey. In Australian Transport Research Forum.

[16] Gong, X., Currie, G., Liu, Z., and Guo, X. (2017). A Disaggregate Study of Urban Rail Transit Feeder Transfer Penalties Including Weather Effects. Transportation, 1-31.

[17] Guan, H.Z., (2004) Disaggregate Model - a traffic behavior analysis tool [M], People's Communications Press.

[18] Jollife I.T. (1986) Principal Component Analysis [M]. New York: Princeton University Press, 56-60. 